\title{
Prevenção do defeito cognitivo e demência - os factores de risco vascular
}

Ana Verdelho*

\section{RESUMO}

O declínio cognitivo e a demência aumentam com o envelhecimento. Por outro lado, os factores de risco vascular também são mais frequentes no indivíduo idoso. Nos últimos anos tem surgido evidência de que os factores de risco vascular contribuem para o declínio cognitivo e a demência. Este efeito tem sido descrito não só para a demência vascular, mas também para as demências degenerativas, nomeadamente para a doença de Alzheimer. O impacto dos diferentes factores de risco na futura evolução para um declínio cognitivo é de avaliação complexa, uma vez que por norma os factores de risco não existem de forma isolada em cada indivíduo, e co-existem com outras variáveis (educacionais, socio-culturais, comportamentais e de estilos de vida) que podem modificar a evolução para declínio cognitivo. Discutem-se nesta secção os dados existentes em relação a vários factores de risco vascular e ainda a comportamentos e estilos de vida que frequentemente se associam aos factores de risco vascular. Perante a evidência actual propõe-se uma estratégia de actuação preventiva.

Palavras-chave: Declínio Cognitivo; Demência e Factores de Risco Vascular.

\section{INTRODUÇÃO}

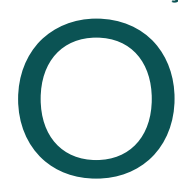

declínio cognitivo e as demências são mais frequentes nos indivíduos idosos. Por outro lado, os factores de risco vascular também são mais frequentes nos indivíduos idosos. Numa época em que assistimos ao envelhecimento populacional e em que a esperança média de vida aumenta consideravelmente, as estratégias para a prevenção do declínio cognitivo e da demência tornam-se uma prioridade no âmbito da saúde pública.

Tradicionalmente havia a convicção de que os factores de risco vascular se associavam à demência de tipo vascular. Nesta patologia, a estratégia é muito consensual: a prevenção dos factores de risco vascular é o passo fundamental para impedir a progressão da doença vascular, e, por outro lado, pode assistir-se a melhorias globais e cognitivas pelo correcto manuseio dos factores de risco vascular nos doentes com defeito cognitivo de causa vascular ou com demência vascular.

No entanto, quanto às demências degenerativas, a informação é menos convincente. Nos últimos anos, es-

*Departamento de Neurociências, Hospital de Santa Maria, Faculdade de Medicina e Instituto de Medicina Molecular, Universidade de Lisboa. pecialmente na última década, os resultados dos estudos epidemiológicos observacionais têm sugerido uma contribuição dos factores de risco vascular no declínio cognitivo e nas demências degenerativas, nomeadamente na doença de Alzheimer. No entanto, os resultados não têm sido consistentes. $\mathrm{O}$ mecanismo pelo qual os factores de risco vascular aumentam o risco de doença de Alzheimer ou aceleram a sua progressão não é claro. A maior parte das hipóteses ressalta o papel de lesões subcorticais, que podem ser avaliadas por exames imagiológicos, tais como as alterações da substância branca, enfartes lacunares ou micro-hemorragias cerebrais. Uma teoria possível seria que existisse um efeito aditivo ou sinergístico das alterações degenerativas típicas da doença de Alzheimer com a patologia vascular, sobretudo pela patologia de pequenos vasos subcortical, e não necessariamente pelos acidentes vasculares hemisféricos. ${ }^{1,2}$ Neste sentido, o controlo e prevenção dos factores de risco vascular (que está implicada na génese da patologia vascular de pequenos vasos) seria uma estratégia possível na prevenção do desenvolvimento e da progressão do declínio cognitivo. No entanto, os ensaios clínicos efectuados com fármacos para controlo de factores 
de risco vascular não têm tido resultados consistentes na prevenção de declínio cognitivo. Um dos aspectos a considerar é que os estudos realizados até à data na prevenção de factores de risco vascular não têm focado especificamente a incidência de doença de Alzheimer como objectivo primário. Por outro lado, os vários estudos podem ter um problema de poder estatístico, não se encontrando uma relação de causalidade entre factor de risco vascular e a demência de Alzheimer por não terem existido observações em número suficiente, nos diferentes estudos, para se verificar um efeito estatisticamente significativo. Um estudo muito recente mostrou que em pacientes com doença de Alzheimer sem evidência de lesões vasculares cerebrais significativas (definidas como ter tido clinicamente um acidente vascular cerebral, ou ter lesões vasculares estratégicas, múltiplas lesões vasculares ou lesões difusas da substância branca cerebral nos exames de imagem), o tratamento dos factores de risco vascular diminuiu a perda de pontos no MMSE. ${ }^{3}$ Há ainda um aspecto que importa mencionar: mesmo que um dado factor de risco vascular provoque um aumento de risco que seja pequeno, se ele for frequente na população, o seu controlo poderá ter um impacto considerável.

Uma outra questão que se coloca quando se avalia o impacto dos factores de risco vascular no declínio cognitivo é que não é possível abordá-los de forma isolada, uma vez que os mesmos se associam a aumento de risco de outras patologias, que, de forma indirecta, podem ter uma repercussão na função cognitiva. Tomando como exemplo o acidente vascular cerebral, este é em si é um factor de risco vascular de demência, mas que se associa a um conjunto de outros factores de risco vascular como a hipertensão, a diabetes ou as dislipidémias.

Procura-se com o presente artigo fazer uma síntese crítica e com utilidade prática da evidência existente sobre o impacto dos factores de risco vascular na evolução para declínio cognitivo e demência, e da estratégia possível para a prevenção dos mesmos.

\section{A HIPERTENSÃO ARTERIAL}

Desde há 20 anos que tem sido descrito um aumento de risco de defeito cognitivo e de demência em indivíduos hipertensos..$^{4-6}$ No entanto, existem também estudos com resultados negativos ou que sugerem que uma pressão diastólica muito baixa poderia aumentar o ris- co. Uma das hipóteses explicativas da controversa relação entre hipertensão e demência é que a relação mude com o tempo e com o estádio cognitivo. Parece existir uma relação protectora da terapêutica da hipertensão efectuada na vida adulta (meia idade). ${ }^{4,6}$ Mais tarde, quando existe já defeito cognitivo, a redução de tensão arterial poderá já não ter o mesmo papel protector. ${ }^{7,8}$ Uma revisão sistemática do efeito da terapêutica anti-hipertensiva no declínio cognitivo não conseguiu encontrar benefício. ${ }^{9}$ No entanto, esta revisão só conseguiu incluir quatro ensaios, que eram demasiado heterogéneos entre si e com um elevado número de doentes que fez abandono terapêutico, tendo encontrado muita dificuldade na análise dos dados. Numa outra revisão sistemática verificou-se que as diferenças na definição de «hipertensão» entre os vários estudos impossibilitavam uma leitura comparativa dos resultados. $^{10}$

\section{A DIABETES}

Tem havido crescente evidência de aumento de risco de defeito cognitivo e demência, incluindo da doença de Alzheimer, em doentes diabéticos. ${ }^{11-13}$ Os estudos observacionais indicam que indivíduos diabéticos têm o dobro do risco de desenvolver defeito cognitivo ligeiro e demência, comparando com aqueles que não têm diabetes. ${ }^{14}$ Inicialmente a convicção era que a diabetes se associaria sobretudo à demência e defeito cognitivo de causa vascular, mas a evidência mais recente sugere que também esta associação também se verifica com a doença de Alzheimer. ${ }^{15}$ Note-se que o efeito da diabetes não parece ser mediado apenas pela lesão cerebral vascular, mas parece haver crescente evidência de que a insulina também tem um papel na patologia da doença de Alzheimer, e que está envolvida no metabolismo da proteína beta-amilóide e da proteína tau. ${ }^{16}$ Mesmo em indivíduos sem demência, os diabéticos têm pior desempenho em diversos testes cognitivos quando comparados com não diabéticos. O nosso grupo encontrou que numa população de idosos independentes e sem demência, com alterações da substância branca cerebral, os doentes com diabetes tinham pior desempenho nos testes que mediam desempenho global, testes de funções executivas, velocidade e controlo motor, atenção e memória, quando comparados com idosos não diabéticos. ${ }^{17}$ Após 3 anos de seguimento a dia- 
betes manteve-se como factor independente predizendo declínio cognitivo e demência. ${ }^{18}$ No entanto, tal como em relação à hipertensão, os estudos têm mostrado resultados discordantes: recentemente um estudo mostrou que, numa população de pacientes com doença de Alzheimer, aqueles que tinham diabetes registaram um menor declínio cognitivo quando comparados com os doentes sem diabetes. ${ }^{19}$ Uma das explicações possíveis é que nos doentes diabéticos se tenha efectuado um controlo mais rigoroso dos factores de risco vascular e implementado terapêuticas (anti-agregantes, hipotensoras e hipolipemiantes) que não tenham sido introduzidas nos doentes sem diabetes. $\mathrm{O}$ outro problema deste estudo é que apenas cerca de $2 / 3$ da amostra inicial completou o seguimento.

Ao longo dos estudos observacionais, o efeito da diabetes foi mais marcado quando foi analisado em conjunto com outros factores de risco, ${ }^{20,21} \mathrm{o}$ que sugere um papel aditivo entre os vários factores de risco vascular, e associou-se à progressão da doença de Alzheimer. ${ }^{21}$

\section{O TABACO}

O efeito do tabaco tem sido também discutido como potencial implicado no declínio cognitivo e demência. O tabaco poderia ter efeito benéfico mediado pela acção estimulante da nicotina. A administração aguda de nicotina em adultos jovens não fumadores com defeito de atenção pareceu melhorar a atenção, as funções executivas e a memória de trabalho, provavelmente através da activação do sistema colinérgico. ${ }^{22}$ No entanto, o efeito nefasto do tabaco, mediado pelo stress oxidativo, a inflamação e promoção da aterogénese podem mediar um aumento de risco de declínio cognitivo, ainda que indirecto.

Recentemente, um estudo efectuado num grupo de idosos de Taiwan mostrou um papel benéfico do tabaco. ${ }^{23}$ No entanto, uma meta-análise de 19 estudos observacionais prospectivos encontrou que o tabaco constituía um factor de risco para demência, e o efeito não se limitava à demência vascular, mas abrangia também as demências degenerativas. ${ }^{24}$ Por outro lado, os resultados foram muito claros na comparação entre fumadores activos e indivíduos que nunca fumaram. $\mathrm{O}$ risco de declínio cognitivo e demência não foi evidente para o grupo de ex-fumadores, o que se prende provavelmente com a marcada variabilidade deste grupo (quer no tempo de contacto com o hábito quer na quantidade de exposição durante esse período).

\section{A DOENÇA VASCULAR CEREBRAL O AVC}

O AVC (quer isquémico quer hemorrágico) tem sido associado a demência subsequente em alguns estudos prospectivos comunitários. ${ }^{26-28} \mathrm{~A}$ associação é encontrada não apenas com a demência vascular, ${ }^{29}$ mas também com a demência degenerativa. Uma revisão sistemática muito recente mostrou que o acidente vascular cerebral duplica o risco de demência (sem especificar o tipo), um aumento que não parece ser só justificado pelos factores de risco cerebrais associados, e que este aumento de risco se perde nos indivíduos muito idosos..$^{30} \mathrm{Uma}$ das explicações possíveis é que o AVC poderá antecipar a manifestação de um declínio cognitivo que estaria já em curso, podendo ser o precipitante de um doente com defeito cognitivo ligeiro passar a ter critérios de demência. Um dos aspectos a realçar neste caso é que a intervenção muito activa, com o objectivo de prevenir uma nova recorrência e complementada com um programa de reabilitação, mesmo em doentes com defeito cognitivo prévio, pode ter um impacto relevante, configurando uma verdadeira possibilidade de recuperação.

\section{As alterações da substância branca cerebral}

As alterações da substância branca cerebral são frequentes em idosos com e sem declínio cognitivo. O nosso grupo encontrou uma relação, consistente com estudos prévios, entre alterações de substância branca severas e idade, AVC lacunar e hipertensão arterial. ${ }^{31}$ Neste mesmo trabalho, observámos que as alterações da substância branca severas se associavam (em indivíduos idosos autónomos e sem demência), a pior desempenho cognitivo global, em funções executivas, atenção e velocidade motora, de acordo com resultados de outros estudos. ${ }^{17,32}$ No seguimento desta mesma população de idosos ao longo de três anos, confirmámos que as alterações severas da substância branca são um factor de mau prognóstico para declínio cognitivo ao longo do tempo (demência e não demência). ${ }^{18}$

\section{OUTROS}

Quanto ao controlo da hipercolesterolémia e da hipertrigliceridémia, vale a pena mencionar que a controvér- 
sia já descrita em relação à diabetes e à hipertensão se verifica novamente, mas existe menor consistência de que a hipercolesterolémia seja um factor de risco isolado de demência de Alzheimer. ${ }^{33}$

Alguns estudos relacionam a obesidade na idade média da vida com um aumento de risco para demência. ${ }^{34}$ No entanto, os doentes com demência tendem a ter menor índice de massa corporal do que indivíduos não dementes, o que poderá traduzir apenas a consequência da própria demência, ou levantar a dúvida de uma relação entre ambos.

Alguns estudos têm ainda abordado o impacto da chamada «síndroma metabólica» (que consiste na conjugação de um conjunto de patologias como a hipertensão, obesidade abdominal, hipertrigliceridémia, baixo nível de lipoproteínas de elevada densidade, e/ou hiperglicémia) no aumento de risco de defeito cognitivo, sobretudo em doentes com identificação de factores inflamatórios.

Os dados em relação ao consumo de bebidas alcoólicas são novamente controversos. O estudo de Roterdão sugeriu que o consumo ligeiro ou moderado de bebidas alcoólicas se associava a uma redução do risco de demência. ${ }^{35}$ Foi descrita uma associação entre atrofia cerebral e consumo de bebidas alcoólicas mesmo para bebedores ligeiros, ${ }^{36}$ mas este estudo não fez avaliação cognitiva dos indivíduos incluídos. No nosso estudo verificámos que indivíduos idosos independentes com consumo etílico ligeiro a moderado tinham desempenho cognitivo ligeiramente melhor, mas este benefício deixou de se verificar durante o seguimento. ${ }^{17}$

\section{VARIÁVEIS ASSOCIADAS AO COMPORTAMENTO E ESTILOS DE VIDA}

Vários estudos têm abordado a dieta como um factor relevante na prevenção de demência. Adicionalmente, muitos dos factores de risco vascular podem ser modificados pela dieta. Na última década, vários estudos sugeriram um papel protector da dieta mediterrânica quanto ao risco de defeito cognitivo ligeiro e de doença de Alzheimer, ${ }^{37,38}$ como factor protector de progressão de defeito cognitivo ligeiro para doença de Alzheimer, ${ }^{38}$ e estando associada a menor mortalidade em doentes com doença de Alzheimer. ${ }^{39}$ A associação entre dietas ricas em peixe, fruta e vegetais e menor risco de demência tem sido atribuída aos anti-oxidantes e aos ácidos gordos poli-insaturados, mas a dose necessária a esta protecção não é clara. Por outro lado, os resultados dos estudos observacionais não têm sido consistentes e os ensaios efectuados com suplementos vitamínicos (incluindo vitamina E e C) e ácidos gordos poli-insaturados não tiveram resultados convincentes.

Outro aspecto da dieta que tem sido investigado é o papel da cafeína como protector de demência. A cafeína tem um efeito estimulante do sistema nervoso central a curto termo, mas o seu impacto como protector a longo termo continua controverso. O consumo de café associa-se a um conjunto de variáveis (sociodemográficas, estilos de vida, alimentares, e mesmo de factores de risco), que por si também influenciam a capacidade cognitiva. Por outro lado, torna-se difícil diferenciar se a predisposição para tomar café é por si parte de uma personalidade que protege de futuro defeito cognitivo, ou se é o café que tem um papel verdadeiramente protector de defeito cognitivo e demência. Outro aspecto é a quantidade necessária à prevenção e a variação em função do género. Um estudo efectuado em vários países europeus, que avaliou apenas o género masculino, sugeriu um efeito protector. ${ }^{40}$ No estudo das três cidades, o efeito protector foi apenas observado no género feminino, ${ }^{41} \mathrm{e}$ em ambos os estudos o benefício foi verificado para cerca de três chávenas/dia. Recentemente, um estudo efectuado na população finlandesa mostrou que o consumo de cafeína na idade adulta (idade média de 50 anos) se associou a menor risco de desenvolver doença de Alzheimer cerca de 21

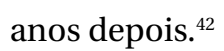

\section{ACTIVIDADE FÍSICA E DE LAZER}

Vários estudos mostraram que a actividade física pode atrasar ou prevenir o declínio funcional associado ao envelhecimento e promover a saúde de forma global. Por outro lado, a participação em actividades de lazer, físicas e cognitivas, parece de forma semelhante ter um papel preventivo na incidência e progressão da demência, incluindo a demência de Alzheimer. ${ }^{43,44-47}$ No entanto, a explicação da relação entre o exercício físico e menor declínio cognitivo e demência não é clara. ${ }^{43,48}$ Uma das razões é que não efectuar exercício físico pode já resultar de um declínio cognitivo muito incipiente, ou derivar de um conjunto de patologias que por si também aumentem o risco de demência, ou ainda en- 
quadrar-se num estilo de vida menos favorável. Um estudo muito recente mostrou uma redução no risco de desenvolver doença de Alzheimer em idosos previamente sem queixas cognitivas, com uma relação dependente da dose, ou seja, o benefício foi registado mesmo para uma pequena quantidade de exercício físico, e seria tanto melhor quanto maior a actividade. ${ }^{49}$

Vale a pena referir que, em relação aos estudos publicados que analisam o efeito do exercício físico no risco de declínio cognitivo e de demência, existe uma diferença considerável em relação aos restantes factores de risco mencionados: nos estudos efectuados registou-se um efeito benéfico ou não se verificou efeito, mas em nenhum se encontrou um aumento de risco de defeito cognitivo ou demência por ter uma actividade física ou de lazer.

\section{NOTA FINAL}

Actualmente, a terapêutica farmacológica das demências consegue apenas melhorar de forma modesta os sintomas e não pode curar ou prevenir a demência, nem tão pouco impedir a progressão de um estado de defeito cognitivo ligeiro para um estado de demência. Como resultado, a identificação de factores de risco para defeito cognitivo e demência e o seu controlo constitui uma estratégia de relevo.

Tradicionalmente, as demências vasculares e de Alzheimer eram vistas como entidades claramente distintas. Progressivamente, tem-se reconhecido que estas patologias raramente ocorrem de forma isolada. Ambas as doenças podem partilhar factores de risco vascular e aspectos anatomo-patológicos de arterios-

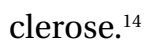

De forma geral, e apesar das controvérsias das publicações, a convicção é que os factores de risco vascular se associam a um aumento de risco de demência vascular e potencialmente também de doença de Alzheimer. A preocupação actual abarca a necessidade de conhecer a contribuição relativa de cada factor de risco vascular para cada um dos tipos de demência e que tipo de associação entre factores de risco vascular potencia o aumento de risco de demência. De forma directa, ou indirecta, os factores de risco vascular, sobretudo se em associação (nomeadamente no caso da hipertensão, da diabetes e do AVC) parecem ser factores de risco também para declínio cognitivo e demência, e não só para morte de causa vascular. Os resultados dos ensaios clínicos não demonstraram de forma convincente que o controlo dos factores de risco vascular previne o desenvolvimento de demência. Uma das razões principais é que existe falta de ensaios desenhados adequadamente, com o objectivo específico de prevenir declínio cognitivo/demência e centrados no controlo dos factores de risco vascular. Note-se que não ter encontrado evidência de uma dada intervenção para prevenção de uma doença, mesmo por meta-análises e revisões sistemáticas, não significa que esta tenha sido ineficaz. Pode apenas traduzir a falta de poder estatístico para se terem tirado conclusões positivas, ou ser o resultado de uma metodologia menos adequada ao objectivo proposto.

De momento, com o objectivo (ainda que possamos admitir indirecto em alguns casos) de prevenção de declínio cognitivo e de demência, a melhor estratégia deverá incluir: 1) identificação dos factores de risco vascular na população em geral, ao longo do envelhecimento; 2) optimização da terapêutica dos factores de risco vascular, com especial incidência na hipertensão, diabetes e acidentes vasculares cerebrais; 3 ) promoção de um estilo de vida saudável, que integre a prática de uma actividade física regular; 4) vigilância regular de queixas cognitivas em indivíduos com factores de risco vascular, tomando especial cuidado em indivíduos com associação de vários factores de risco.

\section{BIBLIOGRAFIA}

1. Vermeer SE, Prins ND, den Heijer T, Hofman A, Koudstaal PJ, Breteler $M M$. Silent brain infarcts and the risk of dementia and cognitive decline. N Engl J Med 2003 Mar 27; 348 (13): 1215-22.

2. Schneider JA, Boyle PA, Arvanitakis Z, Bienias JL, Bennett DA. Subcortical infarcts, Alzheimer's disease pathology, and memory function in older persons. Ann Neurol 2007 Jul; 62 (1): 59-66.

3. Deschaintre $Y$, Richard F, Leys D, Pasquier F. Treatment of vascular risk factors is associated with slower decline in Alzheimer disease. Neurology 2009 Sep 1; 73 (9): 674-80.

4. Launer LJ, Ross GW, Petrovitch H, Masaki K, Foley D, White LR, et al. Midlife blood pressure and dementia: the Honolulu-Asia aging study. Neurobiol Aging 2000 Jan-Feb; 21 (1): 49-55.

5. Ruitenberg A, Skoog I, Ott A, Aevarsson O, Whiteman JC, Lernfelt B, et al. Blood pressure and risk of dementia: results from the Rotterdam study and the Gothenburg H-70 Study. Dement Geriatr Cogn Disord 2001 Jan-Feb; 12 (1): 33-9.

6. Kivipelto M, Helkala EL, Laakso MP, Hänninen T, Hallikainen M,Alhainen $\mathrm{K}$, et al. Midlife vascular risk factors and Alzheimer's disease in later life: longitudinal, population based study. BMJ 2001; 322 (7300): 1447-51. 
7. Shah RC, Wilson RS, Bienias JL, Arvanitakis Z, Evans DA, Bennett DA. Relation of blood pressure to risk of incident Alzheimer's disease and change in global cognitive function in older persons. Neuroepidemiology 2006; 26 (1): 30-6.

8. van Bruchem-Visser RL, Mattace-Raso FU, van der Cammen TJ. High systolic and pulse pressure levels are associated with better cognitive performance in patients with probable Alzheimer's disease: a cross-sectional observational study in a geriatric outpatient population. Dement Geriatr Cogn Disord 2009; 28 (4): 320-4.

9. McGuinness B, Todd S, Passmore P, Bullock R. The effects of blood pressure lowering on development of cognitive impairment and dementia in patients without apparent prior cerebrovascular disease. Cochrane Database Syst Rev 2006 Apr 19; (2): CD004034.

10. Purnell C, Gao S, Callahan CM, Hendrie HC. Cardiovascular risk factors and incident Alzheimer disease: a systematic review of the literature. Alzheimer Dis Assoc Disord 2009 Jan-Mar; 23 (1): 1-10.

11. Biessels GJ, Staekenborg S, Brunner E, Brayne C, Scheltens P. Risk of dementia in diabetes mellitus: a systematic review. Lancet Neurol 2006 Jan; 5: 64-74.

12. Arvanitakis Z, Wilson RS, Bienias JL, Evans DA, Bennett DA. Diabetes mellitus and risk of Alzheimer disease and decline in cognitive function. Arch Neurol 2004 May; 61 (5): 661-6.

13. Cukierman T, Gerstein HC, Williamson JD. Cognitive decline and dementia in diabetes: systematic overview of prospective observational studies. Diabetologia 2005 Dec; 48 (12): 2460-9.

14. Launer LJ. Demonstrating the case that $A D$ is vascular disease: epidemiological evidence. Ageing Res Rev 2002 Feb; 1 (1): 61-77.

15. Luchsinger JA. Adiposity, hyperinsulinemia, diabetes and Alzheimer's disease: an epidemiological perspective. Eur J Pharmacol 2008 May; 585 (1): 119-29.

16. Liu F, Shi J, Tanimukai H, Gu J, Gu J, Grundke-lqbal I, et al. Reduced OGlcNAcylation links lower brain glucose metabolism and tau pathology in Alzheimer's disease. Brain 2009 Jul; 132 (Pt 7): 1820-32.

17. Verdelho A, Madureira S, Ferro JM, Basile AM, Chabriat H, Erkinjuntii T, et al. Differential impact of cerebral white matter changes, diabetes, hypertension and stroke on cognitive performance among non-disabled elderly. The LADIS study. J Neurol Neurosurg Psychiatr.2007 Dec; 78 (12): 1325-30.

18. Verdelho A, Madureira S, Moleiro C, Ferro JM, Santos CO, Erkinjuntti T, et al. White matter changes and diabetes predict cognitive decline in the elderly: the LADIS study [in press].

19. Sanz C, Andrieu S, Sinclair A, Hanaire H, Vellas B; REAL.FR Study Group. Diabetes is associated with a slower rate of cognitive decline in Alzheimer disease. Neurology 2009 Oct; 73 (17): 1359-66.

20. Luchsinger JA, Reitz C, Honig LS, Tang MX, Shea S, Mayeux R. Aggregation of vascular risk factors and risk of incident Alzheimer disease. Neurology 2005 Aug 23; 65 (4): 545-51.

21. Helzner EP, Luchsinger JA, Scarmeas N, Cosentino S, Brickman AM, Glymour $\mathrm{MM}$, et al. Contribution of vascular risk factors to the progression in Alzheimer disease. Arch Neurol 2009 Mar; 66 (3): 343-8.

22. Potter AS, Newhouse PA. Acute nicotine improves cognitive deficits in young adults with attention-deficit/hyperactivity disorder. Pharmacol Biochem Behav 2008 Feb;88 (4): 407-17.

23. Wang CC, Lu TH, Liao WC, Yuan SC, Kuo PC, Chuang HL, et al. Cigarette smoking and cognitive impairment: a 10-year cohort study in Taiwan. Arch Gerontol Geriatr 2009 Oct 13. [Epub ahead of print]. doi:10.1016/j.archger.2009.09.041

24. Anstey KJ, von Sanden C, Salim A, O'Kearney R. Smoking as a risk factor for dementia and cognitive decline: a meta-analysis of prospective studies. Am J Epidemiol 2007 Aug 15; 166 (4): 367-78.

26. Rastas S, Pirttilä T, Mattila K, Verkkoniemi A, Juva K, Niinistö L, et al. Vascular risk factors and dementia in the general population aged $>85$ years: prospective population-based study. Neurobiol Aging 2010 Jan; 31 (1): 1-7.

27. Reitz C, Bos MJ, Hofman A, Koudstaal PJ, Breteler MM. Prestroke cognitive performance, incident stroke, and risk of dementia: the Rotterdam Study. Stroke 2008 Jan; 39 (1): 36-41.

28. Yip AG, Brayne C, Matthews FE; MRC Cognitive Function and Ageing Study. Risk factors for incident dementia in England and Wales: The Medical Research Council Cognitive Function and Ageing Study: a population-based nested case-control study. Age Ageing 2006 Mar; 35 (2): 154-60.

29. Hénon H, Pasquier F, Leys D. Poststroke dementia. Cerebrovasc Dis 2006; 22 (1): 61-70.

30. Savva GM, Stephan BC; Alzheimer's Society Vascular Dementia Systematic Review Group. Epidemiological studies of the effect of stroke on incident dementia: a systematic review. Stroke 2010 Jan; 41 (1): e41-6.

31. Basile AM, Pantoni L, Pracucci G, et al. Age, hypertension, and lacunar stroke are the major determinants of the severity of age-related white matter changes. The LADIS (Leukoaraiosis and Disability in the Elderly) Study. Cerebrovasc Dis 2006; 21 (5-6): 315-22.

32. Prins ND, van Dijk EJ, den Heijer T, Vermeer SE, Jolles J, Koudstaal PJ, et al. Cerebral small-vessel disease and decline in information processing speed, executive function and memory. Brain 2005 Sep; 128 (Pt 9): 2034-41.

33. Stefani M, Liguri G. Cholesterol in Alzheimer's disease: unresolved questions. Curr Alzheimer Res 2009 Feb; 6 (1): 15-29.

34. Hughes TF, Borenstein AR, Schofield E, Wu Y, Larson EB. Association between late-life body mass index and dementia: the Kame Project. Neurology 2009 May 19; 72 (20): 1741-6.

35. Ruitenberg A, van Swieten JC, Witteman JC, Mehta KM, van Duijn CM, Hofman A, et al. Alcohol consumption and risk of dementia: the Rotterdam Study. Lancet 2002 Jan 26; 359(9303): 281-6.

36. Ding J, Eigenbrodt ML, Mosley TH Jr, Hutchhinson RG, Folsom AR, Harris TB, et al. Alcohol intake and cerebral abnormalities on magnetic resonance imaging in a community-based population of middle-aged adults: the Atherosclerosis Risk in Communities (ARIC) study. Stroke 2004 Jan; 35 (1):16-21.

37. Scarmeas N, Stern Y, Tang MX, Mayeux R, Luchsinger JA. Mediterranean diet and risk for Alzheimer's disease. Ann Neurol 2006 Jun; 59 (6): $912-$ $-21$.

38. Scarmeas N, Stern Y, Mayeux R, Manly JJ, Schupf N, Luchsinger JA. Mediterranean diet and mild cognitive impairment. Arch Neurol 2009 Feb; 66 (2): 216-25.

39. Scarmeas N, Luchsinger JA, Mayeux R, Stern Y. Mediterranean diet and Alzheimer disease mortality. Neurology 2007 Sep 11; 69 (11): 1084$-93$.

40. van Gelder BM, Buijsse B, Tijhuis M, Kalmijn S, Giampaoli S, Nissinen A, 
et al. Coffee consumption is inversely associated with cognitive decline in elderly European men:The FINE Study. Eur J Clin Nutr 2007 Feb; 61 (2): 226-32.

41. Ritchie $K$, Carrière I, de Mendonca A, Portet F, Dartigues JF, Rouaud O, et al. The neuroprotective effects of caffeine: a prospective population study (the Three City Study). Neurology 2007 Aug 7; 69 (6):, 536-45.

42. Eskelinen MH, Ngandu T, Tuomilehto J, Soininen H, Kivipelto M. Midlife coffee and tea drinking and the risk of late-life dementia: a populationbased CAIDE study. J Alzheimers Dis 2009 Jan; 16 (1): 85-91.

43. Verghese J, Lipton RB, Katz MJ, Hall CB, Derby CA, Kuslansly G, et al. Leisure activities and the risk of dementia in the elderly. $\mathrm{N}$ Engl J Med 2003 Jun 19; 348 (25): 2508-16.

44. Larson EB, Wang L, Bowen JD, McCormick WC, Teri L, Crane P, et al. Exercise is associated with reduced risk for incident dementia among persons 65 years of age and older. Ann Intern Med 2006 Jan 17; 144 (2): 73-81.

45. Weuve J, Kang JH, Manson JE, Breteler MM, Ware JH, Grodstein F. Physical activity, including walking, and cognitive function in older women. JAMA 2004 Sep 22; 292 (12): 1454-61.

46. Rovio S, Kareholt I, Helkala EL, Viitanen M, Winblad B, Tuomilehto J, et al. Leisure-time physical activity at midlife and the risk of dementia and Alzheimer's disease. Lancet Neurol 2005 Nov; 4 (11): 705-11.
47. Wang L, Larson EB, Bowen JD, van Belle G. Performance- based physical function and future dementia in older people. Arch Intern Med 2006 May 22; 166 (10): 1115-20.

48. Forbes D, Forbes S, Morgan DG, Markle-Reid M, Wood J, Culum I. Physical activity programs for persons with dementia. Cochrane Database Syst Rev 2008 Jul 16; (3): CD006489.

49. Scarmeas N, Luchsinger JA, Schupf N, Brickman AM, Cosentino S, Tang $M X$, et al. Physical activity, diet, and risk of Alzheimer disease. JAMA 2009 Aug 12; 302 (6): 627-37.

A autora declarou não possuir conflitos de interesses

\section{ENDEREÇO PARA CORRESPONDÊNCIA}

Ana Verdelho

Departamento de Neurociências.Hospital de Santa Maria.

Av. Prof. Egas Moniz. 1649-028 Lisboa.

averdelho@fm.ul.pt

\section{ABSTRACT}

\section{VASCULAR RISK FACTORS AND PREVENTION OF COGNITIVE DECLINE AND DEMENTIA}

Cognitive decline and dementia are frequent in the elderly. Vascular risk factors' frequency also increases with ageing. There has been growing evidence of the implication of vascular risk factors in the evolution for cognitive decline and dementia. This association has been found not only for vascular dementia, but also for degenerative dementias, namely for Alzheimer's disease. The impact of vascular risk factors as predictors for dementia is a complex issue, as they are usually associated with other variables (educational, social, cultural, behavioral and life-style) that may also contribute to the evolution for cognitive decline and dementia. In this section we discuss the association between vascular risk factors, behaviours, and lyfe-style and the evolution for cognitive decline and dementia. We suggest a preventive strategy based on current evidence.

Keywords: Cognitive Decline; Dementia and Vascular Risk Factors. 